\title{
Closing the gap between coil and balloon in the neurointerventional armamentarium? Initial clinical experience with a nitinol vascular occlusion plug
}

\author{
Jan Gralla • Gerhard Schroth • Ralph Kickuth • \\ Marwan El-Koussy • Dai-Do Do • Caspar Brekenfeld
}

Received: 4 February 2008 / Accepted: 8 April 2008 / Published online: 28 May 2008

(C) Springer-Verlag 2008

\begin{abstract}
Introduction The use of vascular plug devices for the occlusion of high-flow lesions is a relatively new and successful procedure in peripheral and cardiopulmonary interventions. We report on the use and efficiency of the Amplatzer vascular plug in a small clinical series and discuss its potential for occlusion of large vessels and highflow lesions in neurointerventions.

Methods Between 2005 and 2007 four patients (mean age 38.5 years, range $16-62$ years) were treated with the device, in three patients to achieve parent artery occlusion of the internal carotid artery, in one patient to occlude a high-flow arteriovenous fistula of the neck. The application, time to occlusion, and angiographic and clinical results and the follow-up were evaluated.

Results Navigation, positioning and detachment of the device were satisfactory in all cases. No flow-related migration of the plug was seen. The cessation of flow was delayed by a mean of $10.5 \mathrm{~min}$ after deployment of the first device. In the procedures involving vessel sacrifice, two devices had to be deployed to achieve total occlusion. No
\end{abstract}

J. Gralla $(\bowtie) \cdot$ G. Schroth $\cdot$ M. El-Koussy $\cdot$ C. Brekenfeld

Department of Diagnostic and Interventional Neuroradiology,

Inselspital, University of Bern,

Freiburgstrasse 20,

CH-3010 Bern, Switzerland

e-mail: jan.gralla@insel.ch

R. Kickuth

Department of Diagnostic, Interventional and Pediatric Radiology,

Bern, Switzerland

D.-D. Do

Department of Angiology, Inselspital, University of Bern,

Bern, Switzerland patient experienced new neurological deficits; the 3-month follow-up revealed stable results.

Conclusion The Amplatzer vascular plug can be adapted for the treatment of high-flow lesions and parent artery occlusions in the head and neck. In this small series the use of the devices was uncomplicated and safe. The rigid and large delivery device and the delayed cessation of flow currently limit the device's use in neurointerventions.

Keywords Amplatzer vascular plug · Vessel sacrifice · Carotid-cavernous fistula $\cdot$ Arteriovenous malformation

\section{Introduction}

The clinical efficiency of the Amplatzer vascular plug (AVP; AGA Medical, Golden Valley, MN) has been described for the occlusion of various pulmonary, coronary and peripheral vessels including in patients with fistulas and arteriovenous malformations in different locations [15]. The transfer of the technology to the field of neurointerventions might therefore be feasible as occlusion of high-flow vascular lesions and sacrifice of large cervical vessels are common neurointerventional procedures. At present, embolic devices such as coils and balloons are predominantly used for these purposes [6-9]. Theoretically, the AVP combines the advantages of a coil with the possibility of resheathing and detaching the device precisely and has the advantage of the straightforward single-step vessel occlusion of a balloon. This embolization approach is relatively new to the neurointerventional field and to date the role of vascular occlusion plugs in these procedures has rarely been documented in the literature $[10,11]$. Specific neuroradiological aspects such as the thromboembolic potential have not been evaluated yet. 
Consequently, to address the need for more data, we evaluated our experience using the AVP in a few different interventions and report the application, safety and efficiency of the device and its potential for occlusion of highflow vessels and lesions.

\section{Methods}

Device design and application

The Amplatzer vascular plug was initially designed for interventions in the peripheral vasculature. The cylindrical device consists of a self-expanding nitinol wire mesh (Fig. 1) and comes in different diameters from $4 \mathrm{~mm}$ to $16 \mathrm{~mm}$ (in 2-mm increments). Selection of a device diameter $30-50 \%$ larger than the vessel is recommended. Each end is radioopacified by a platinum-iridium marker. The device is mounted on a stainless steel delivery wire. The detachment system consists of a microscrew, untightened by clockwise rotation of the wire. Until detachment, it is possible to resheath and redeploy the device to improve positioning. It is possible to control the stability of the position by gentle movement of the delivery wire before definitive detachment. Due to the radial force of the device and the oversized diameter it anchors to the vessel wall. The high friction with the intima does not allow navigating the unsheathed device within the vessel. The device is deployed directly from the guide catheter. Depending on the diameter of the selected device a $5 \mathrm{~F}$ to $8 \mathrm{~F}$ application/ guide catheter is required. At present its use in neurointerventions is "off-label".

\section{Evaluation and intervention}

Since January 2005 four patients (mean age 38.5 years, range 16-62 years) have been treated in our department with the device. In three patients a parent artery occlusion (PAO) of the internal carotid artery (ICA) was performed using the AVP. These patients had a giant aneurysm of the cavernous ICA, a false aneurysm of the cavernous ICA with massive trans-sphenoidal haemorrhage after ENT surgery, a direct carotid-cavernous fistula (CCF) type after head trauma, and a growing, haemodynamically relevant arteriovenous fistula (AVF) of the neck. Angiography and the interventions were performed under general anaesthesia. Intraarterial digital subtraction angiography (DSA) was carried out on a biplane high-resolution angiography system (Toshiba CAS500, Tokyo, Japan). Patient data and details of the interventions carried out are shown in Table 1.

Before PAO detailed DSA to analyse the flow pattern and/or ipsilateral test balloon occlusion to evaluate the intracranial cross-flow were performed. Preinterventional angiography included injection of the common carotid artery (CCA), ICA and external carotid artery (ECA). The extent and supply of the aneurysm, AVF and CCF were documented in multiple projections. To reduce the risk of thromboembolic complications a continuous flush with heparinized sodium chloride solution $(5 \mathrm{IU} / \mathrm{ml})$ was administered. No postprocedural anticoagulation (e.g. aspirin) was administered. In three patients bare platinum coils (Boston Scientific Target, Fremont, CA) were additionally placed. In one patient fibred coils were additionally used to occlude a CCF. Control angiography revealed the flow conditions of the embolized vessel segment and time to occlusion (between the deployment of the first AVP and disruption of flow) was documented. All patients underwent a 3-month follow-up to document the long-term result.

\section{Results}

In all patients the deployment of the AVP led to total occlusion of the vessel/AVF. The AVP were deployed using $6-8 \mathrm{~F}$ guide catheters. In the three patients with $\mathrm{PAO}$ of the ICA, two devices were required to achieve flow arrest. The mean time to total occlusion of the vessel segment after deployment of the first AVP was 10.5 min (range 9$12 \mathrm{~min}$ ). Navigation of the device was limited due to the

Fig. 1 The cylindrical AVP and the positioning and detachment wire (a) and the front view of the dense nitinol mesh (b)
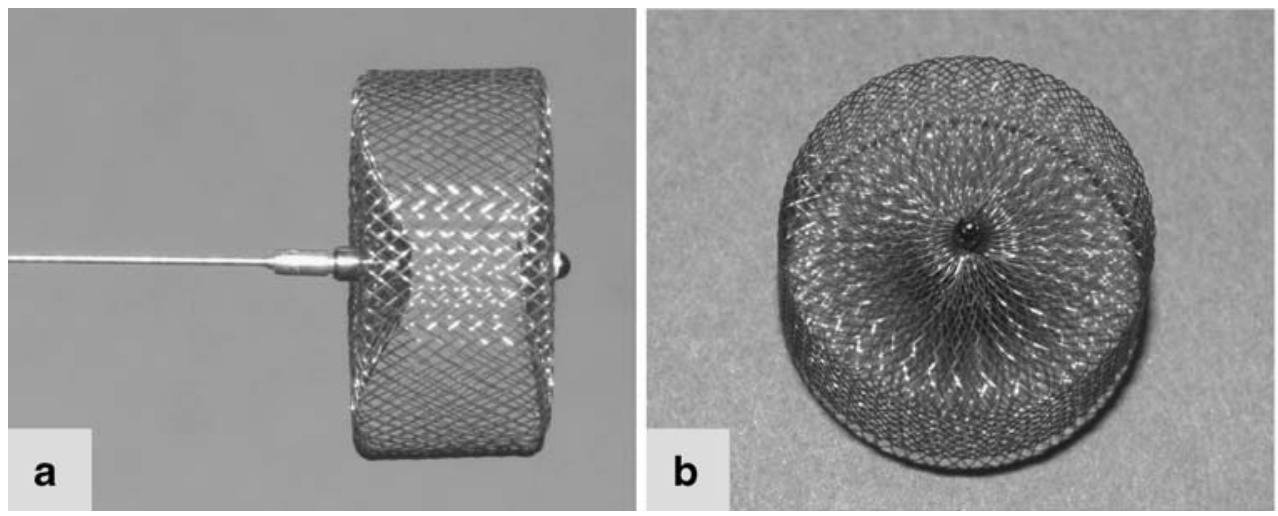
Table 1 Patient data

\begin{tabular}{llllllll}
\hline Age (years) & Gender & Vascular lesion & Vessel occluded & Guide catheter (F) & $\begin{array}{l}\text { No. of AVP } \\
\text { deployed }\end{array}$ & $\begin{array}{c}\text { Additional coils } \\
\text { Time to total } \\
\text { occlusion (min) }\end{array}$ \\
\hline 18 & M & CCF type A & ICA & 7 & 2 & GDC/fibred \\
16 & M & AVF of the neck & Fistula point & 6 & 1 & - & 12 \\
58 & F & ICA giant aneurysm & ICA & 8 & 2 & GDC & 11 \\
62 & M & ICA pseudoaneurysm & ICA & 8 & 2 & GDC \\
\hline
\end{tabular}

rigid and large guide catheter. However, in this limited study in each patient the device was deployed in the target position. The process of detachment was uncomplicated and safe. Once the AVP was detached, no device migration was observed. None of the patients developed new neurological deficits in the postinterventional course. The follow-up revealed stable vessel occlusion in all patients.

Illustrative cases

\section{Patient 1}

An 18-year-old man presented to our emergency department after severe head trauma from a road accident. A cranial CT scan revealed traumatic intracerebral and subarachnoid haemorrhage. Multiple fractures of the scull base involved both pyramids and the sphenoid bone and crossed the carotid canal. Bone fragments were found in the left carotid canal and cavernous sinus. DSA performed 3 days later showed a CCF type A on the left side with the typical draining pattern and dilated cortical veins (Fig. 2). Right carotid and vertebral angiography revealed spontaneous cross-flow to the distal left ICA.

After placement of a $7 \mathrm{~F}$ catheter sheath in the right femoral artery a $7 \mathrm{~F}$ guiding catheter was placed in the cervical segment of the left ICA. Subsequently a microcatheter (FastTracker 18; Boston Scientific Target, Fremont, CA) was advanced and passed into the left cavernous sinus. Due to the extension of the CCF from the distal petrous to the intracavernous segment of the ICA, occlusion of the fistula or preservation of the ICA was not indicated. The target of the intervention was stable occlusion of the ICA proximally and distally to the extended lesion of the vessel wall. Guglielmi detachable coils (GDC) and fibred coils were deployed via the microcatheter in the cavernous segment of the ICA. The control angiogram excluded any residual distal inflow to the fistula from the contralateral side. However, the ipsilateral flow to the fistula was significantly reduced but not disrupted after coil placement. In this specific setting of a partially treated CCF type A an AVP $(8 \mathrm{~mm} \times 7 \mathrm{~mm})$ was navigated and detached in the upper cervical segment of the ICA. Navigation further distally into the petrous segment was abandoned due to the high friction between the device and the vessel wall. After detachment the device led immediately to a further reduction in flow but total cessation was not attained. Combination with a second AVP of the same size placed proximally than achieved total occlusion of the left ICA and therefore the occlusion of the CCF. The 3-month follow-up revealed stable occlusion of the CCF and left ICA.

\section{Patient 2}

A 16-year-old boy presented with a high-flow AVF located at the left side of the neck. The lesion was haemodynamically relevant and had caused a left ventricular enlargement. DSA revealed a markedly enlarged feeder originating from the left ECA (Fig. 3). The fistula (4.5 $\mathrm{mm}$ in diameter) drained directly into the left external jugular vein. No stenosis of the cervical veins was demonstrated. The intracranial venous drainage was not compromised; a cranial MRI scan showed no brain oedema.

After placement of two catheter sheaths in the right femoral artery and femoral vein, both the single feeding ECA branch and the draining vein were catheterized with $6 \mathrm{~F}$ guiding catheters. The venous guide catheter was navigated through the fistula point guided by a biplane road map obtained form arterial injection of contrast agent.

During slow retraction of the catheter into the external jugular vein the $8 \mathrm{~mm} \times 7 \mathrm{~mm}$ AVP was placed in the fistula and subsequently detached. Immediate angiography performed from the arterial catheter showed a marked reduction in flow in the fistula. Ten minutes after detachment of the device the flow in the fistula was disrupted. Consequently, no additional coil treatment had to be performed and the procedure was terminated. Clinical and ultrasound examination after the intervention and after 6 months revealed stable occlusion of the fistula.

The two other patients presented with a giant aneurysm and pseudoaneurysm of the ICA. In both patients the parent artery was occluded after the deployment of two AVP devices through an $8 \mathrm{~F}$ guiding catheter. The distal device was placed into the distal cervical ICA, and navigation into the petrous ICA was not attempted. In both patients placement of a second and proximal device led to total cessation of flow. 
Fig. 2 DSA in an 18-year-old man with a left-sided CCF type A. a, b AP (a) and lateral (b) projections show that the left ICA terminates in the fistula and does not contribute to the intracranial perfusion. c The cavernous sinus drains into the inferior petrosal and pterygoid sinus, and the cortical veins are dilated. d After coil placement the distal inflow zone to the fistula is occluded and the ipsilateral ICA still feeds the fistula. e Two AVP devices are precisely placed and detached within the cervical segment of the ICA. Immediately after placement the flow is reduced at the level of the proximal device (white arrows). $\mathbf{f}$ The ICA is subsequently totally occluded at the level of the distal AVP (black arrows)
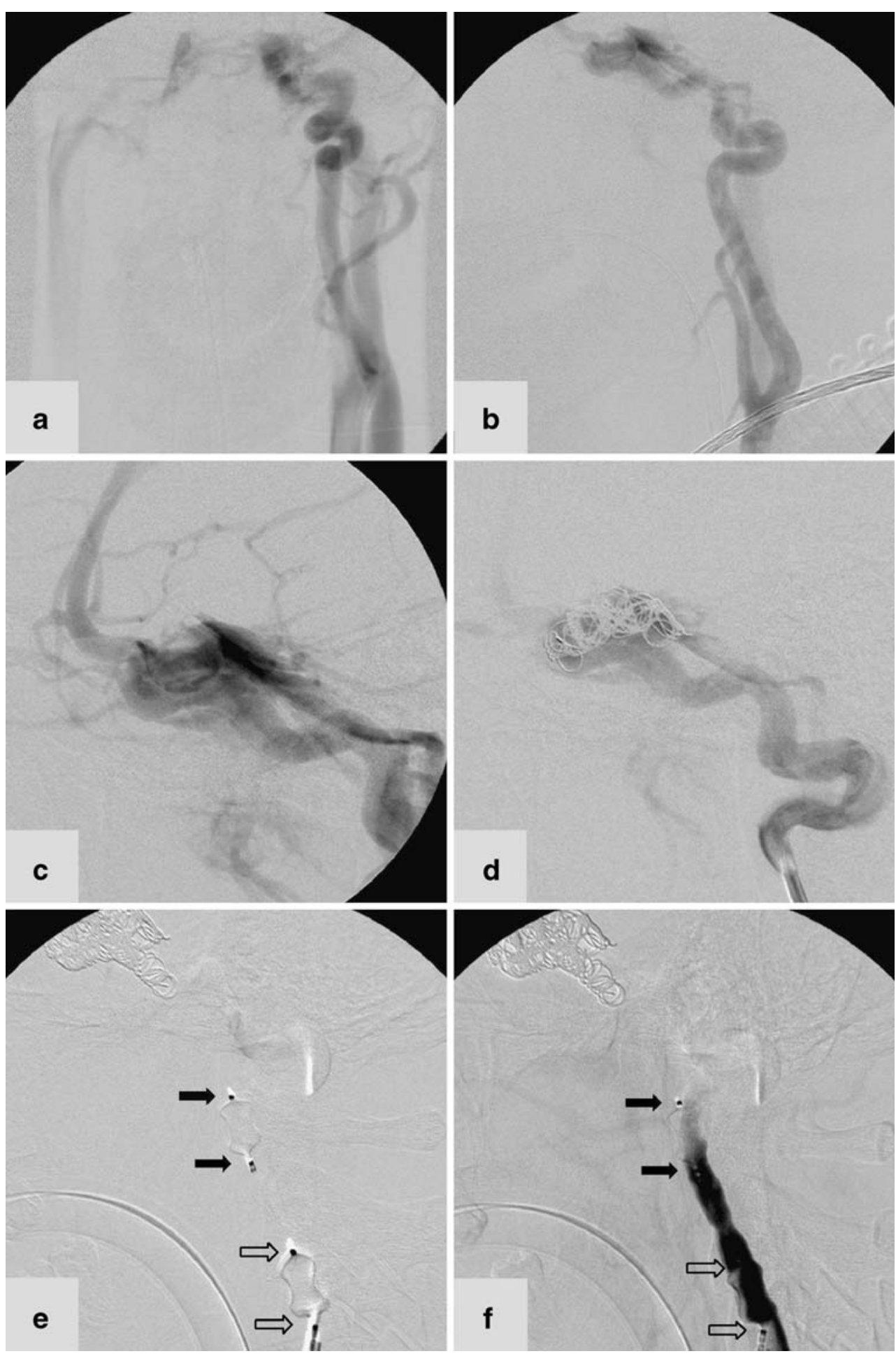

\section{Discussion}

Using a vascular plug device for occlusion of high-flow vascular lesions is an established procedure in peripheral and cardiopulmonary interventions [1-5]. Occlusion of high-flow vascular lesions and sacrifice of large cervical vessels are common neurointerventional procedures, and the recent transfer of the technology to the field of neurointerventions may therefore be a promising development.

At present, endovascular occlusion of these lesions primarily relies on the use of coils and balloons. Coils have the advantage of a very precise placement and detachment. The drawback is the occasionally unstable anchorage at the vessel wall due to the small interface. 

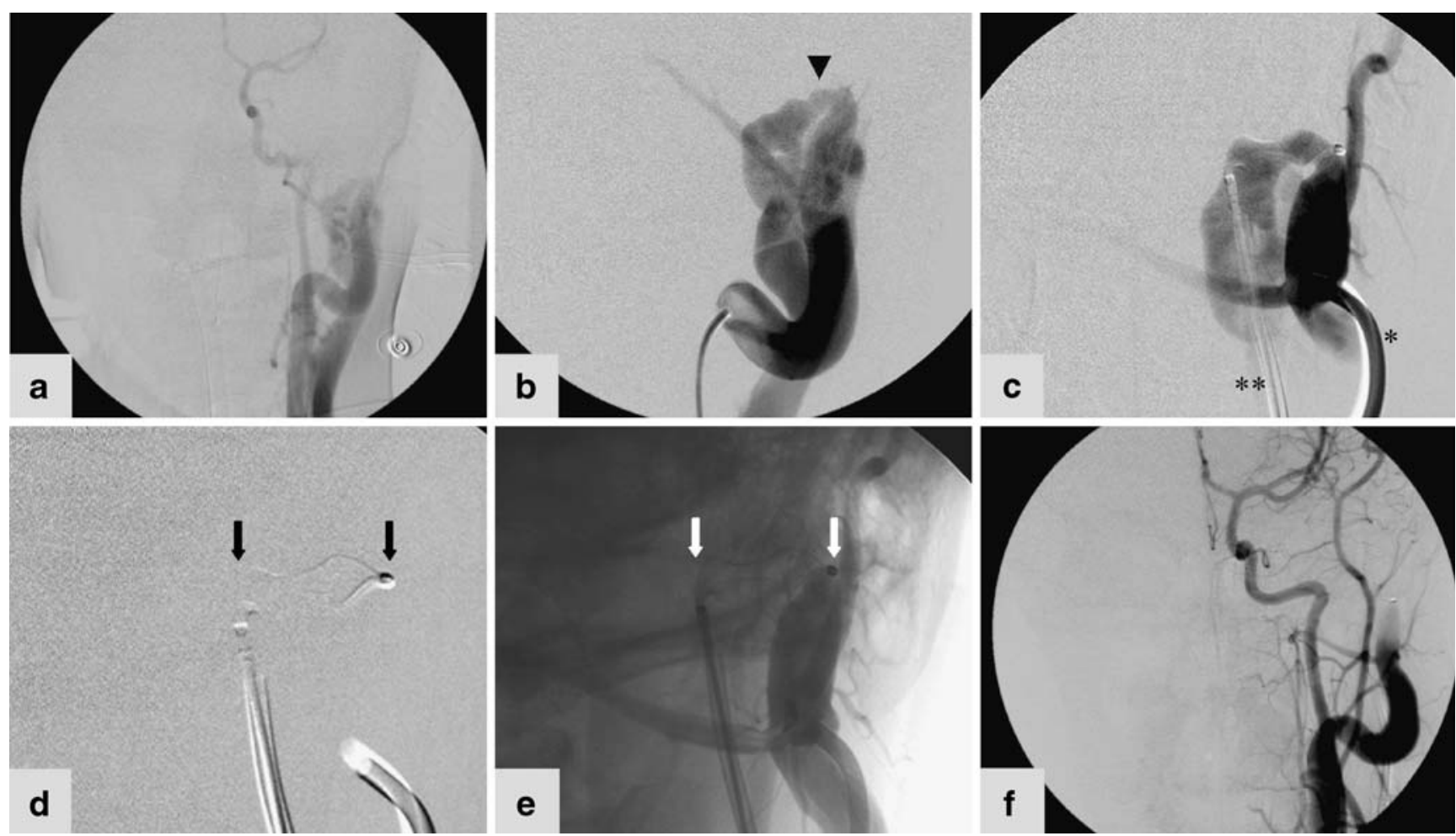

Fig. 3 DSA in a 16-year-old boy with a high-flow AVF. a Left-sided CCA injection reveals the high-flow AVF supplied by the main trunk of the ECA and draining via the internal jugular vein. The AP projection illustrates reduced flow in the ICA territory. b Injection of contrast agent via the arterial guide catheter in the ECA demonstrates the arteriovenous fistula point (arrowhead). c A second catheter is placed in the proximal internal jugular vein (pair of asterisks) in

proximity to the fistula point (single asterisk first catheter). d The AVP device is placed across the fistula point (black arrows) and detached. e The control angiogram reveals total occlusion of the fistula at the arterial border of the device (white arrows). $\mathbf{f}$ The final angiogram demonstrates total occlusion of the fistula and improvement of the flow in the ICA territory

Therefore, the coil ball might be prone to distal migration (e.g. pushable coils or the initially deployed detachable coil). Additionally, the use of multiple coils to occlude an artery is a step-wise, therefore time-consuming, procedure. This time between deployment of the device and total occlusion of the vessel provides an opportunity for the generation thromboembolic complications, and should therefore be minimized in brain-supplying vessels. Some centres use fibred coils [12] or Hydrogel-coated coils (MicroVention, Aliso Viejo, CA) to improve the volumetric filling, reduce the number of coils and accelerate the occlusion process $[13,14]$. Occlusion using coils is, furthermore, a relatively expensive procedure.

A detachable balloon, on the other hand, allows a singlestep occlusion of a vessel or vascular lesion. The risk of thromboembolic events from a clotting process is therefore minimized. Conversely, the deployment of the balloon is challenging and carries the risk of unintentional detachment or early deflation with embolization of the device itself. To prevent early deflation of the balloon, in some centres a hardening embolic material (e.g. a polymer) is used for the final inflation before detachment. This method, however, hinders any further repositioning attempts. The balloon does not anchor itself to the vascular wall and is therefore

prone to flow-induced migration. Therefore the placement of second balloon proximally might be necessary.

The AVP combines some of the advantages of coils and balloons. The navigation of the catheter and the device was uncomplicated in the selected patients including the presented venous approach. The possibility to resheath and reposition the device in combination with a safe and uncomplicated detachment system allowed precise positioning in clinical use. In addition, because of its cylindrical design and high radial force, the device fixes to the vessel wall. No migration of the device was observed clinically. The results in the reported patients were stable during the follow-up period. Furthermore, the device is inexpensive (500 US\$ per device regardless of size) compared to the use of multiple coils.

During our limited clinical experience two aspects emerged as possible limitations. First, the rather rigid delivery wire with the microscrew for detachment limits the navigability of the device in tortuous vessels. In combination with the large guide catheter (minimum 5F) the device is not suitable for use in small-calibre vessels or the intracranial vasculature. Second, the nitinol wire mesh of the AVP significantly reduces flow in the vessel or vascular lesion, even in high-flow lesions as discussed in 
this report. But compared to balloon occlusion, during PAO complete cessation of flow is delayed (mean $10.5 \mathrm{~min}$ ). This delay represents a possible window for thromboembolic complications during PAO during neurointerventions. Consequently, in interventions requiring ICA sacrifice two devices had to be deployed to achieve occlusion in a reasonable time frame $[10,11]$. Both of these limitations indicate the need for adaptation of the device to meet the demands of neurointerventional procedures. A more delicate delivery system would enable the placement in various locations during neuroradiological procedures. The current design seems to hinder intracranial use. Furthermore, a higher efficiency in reducing flow (e.g. denser nitinol mesh or coating) might allow a single-step occlusion and reduce the risk of arterioarterial thromboembolism.

\section{Conclusion}

The AVP can be used for the treatment of high-flow lesions and parent artery occlusions in the head and neck. In this small series the use of the devices was uncomplicated and safe. Its method of placement and the delayed cessation of flow limits its use for neurointerventions.

Conflict of interest statement We declare that we have no conflict of interest.

\section{References}

1. Kickuth R, Dick F, Triller J, Ludwig K, Schmidli J, Do DD (2007) Internal iliac artery embolization before endovascular repair of aortoiliac aneurysms with a nitinol vascular occlusion plug. J Vasc Interv Radiol 18:1081-1087

2. de Giovanni JV (2001) The use of Amplatzer devices to occlude vascular fistulae. J Interv Cardiol 14:45-48

3. Hijazi ZM (2004) New device for percutaneous closure of aortopulmonary collaterals. Catheter Cardiovasc Interv 63:482-485

4. Hill SL, Hijazi ZM, Hellenbrand WE, Cheatham JP (2006) Evaluation of the AMPLATZER vascular plug for embolization of peripheral vascular malformations associated with congenital heart disease. Catheter Cardiovasc Interv 67:113-139

5. Wilkinson JL (2000) Interventional pediatric cardiology: device closures. Indian J Pediatr 67:S30-S36

6. Theaudin M, Saint-Maurice JP, Chapot R, Vahedi K, Mazighi M, Vignal C, Saliou G, Stapf C, Bousser MG, Houdart E (2007) Diagnosis and treatment of dural carotid-cavernous fistulas: a consecutive series of 27 patients. J Neurol Neurosurg Psychiatry 78:174-179

7. Gupta AK, Purkayastha S, Krishnamoorthy T, Bodhey NK, Kapilamoorthy TR, Kesavadas C, Thomas B (2006) Endovascular treatment of direct carotid cavernous fistulae: a pictorial review. Neuroradiology 48:831-839

8. Kirsch M, Henkes H, Liebig T, Weber W, Esser J, Golik S, Kuhne D (2006) Endovascular management of dural carotid-cavernous sinus fistulas in 141 patients. Neuroradiology 48:486-490

9. Jansen O, Dorfler A, Forsting M, Hartmann M, von Kummer R, Tronnier V, Sartor K (1999) Endovascular therapy of arteriovenous fistulae with electrolytically detachable coils. Neuroradiology 41:951-957

10. Hoit DA, Schirmer CM, Malek AM (2006) Use of the Amplatzer vascular plug as an anchoring scaffold for coil-mediated parent vessel occlusion: technical case report. Neurosurgery 59: $171-172$

11. Ross IB, Buciuc R (2007) The vascular plug: a new device for parent artery occlusion. AJNR Am J Neuroradiol 28:385-386

12. Halbach VV, Dowd CF, Higashida RT, Balousek PA, Urwin RW (1998) Preliminary experience with an electrolytically detachable fibered coil. AJNR Am J Neuroradiol 19:773-777

13. Kallmes DF, Cloft HJ (2004) The use of hydrocoil for parent artery occlusion. AJNR Am J Neuroradiol 25:1409-1410

14. Kallmes DF, Fujiwara NH (2002) New expandable hydrogelplatinum coil hybrid device for aneurysm embolization. AJNR Am J Neuroradiol 23:1580-1588 